\title{
Effectiveness of Stretching Exercises in TMJ Closed Lock Management - Case Reports
}

\author{
Marta Miernik* and Agnieszka Nowakowska-Toporowska \\ Department of Prosthodontics, Wroclaw Medical University, Wroclaw, Poland
}

\begin{abstract}
Background: Internal derangement in temporomandibular joint (TMJ), including disc displacement without reduction with limited mouth opening, is a common TMJ disorder. The main symptoms of closed lock are limited mouth opening and pain in the affected TMJ. In such clinical cases, therapeutic management might be administered by physical therapy.

Cases presentation: The first case is a 65-year-old male with closed lock for whom achieving physiological range of mouth opening was crucial due to previously planned cardiac surgery requiring intubation for general anesthesia. The second case is a 15-year-old female looking for immediate medical help caused by restricted mouth opening and pain in TMJ, disturbing her daily routine life.
\end{abstract}

Conclusions: In both presented cases, the range and path of mouth opening were improved. Stretching exercises are an effective method for closed lock management.

\section{Keywords}

TMJ, Closed lock, Stretching exercises

\section{Introduction}

The Axis I of Research Diagnostic Criteria for Temporomandibular Disorders (RDC/TMD) classifies TMD into three main groups: (1) Myofascial pain; (2) Internal derangement of the TMJ; and (3) Arthralgia, osteoarthritis, and osteoarthrosis. The internal derangement occurs as disc displacement with or without reduction. According to the RDC/TMD Axis I Classification, disc displacement without reduction with limited opening, also known as closed lock, is classified as group Ilb [1].

The epidemiological data point that the most common TMD disorders are myofascial pain and TMJ derangements with a prevalence of $45.3 \%$ and $41.1 \%$, respectively. The incidence of disc displacement without reduction with limited opening is estimated to occur in about $5.5 \%$ of TMD patients. Among the cases of closed lock, the anterior displacement is the most common type witha frequency of $61.1 \%$ [2-4].

The etiology of disc displacement is not fully determined, but there are many factors that seem to be related to this disorder, such as anatomical factors, parafunctions, general joint hypermobility, or trauma. The American Academy of Orofacial Pain describes disc displacement without reduction as an incorrect structural relationship between articular disc and condylar head, which is maintained during mandibular translation [5]. The displaced disc is one of the causes of restriction of mandibular movements as it limits the condylar translating movement [6]. The diagnosis of group IIb RDC/
TMD is based on clinical findings and magnetic resonance imaging (MRI). The clinical outcomes include painful limitation in mouth opening ( $\leq 35 \mathrm{~mm}$ ) with passive stretch increasing it by an additional $4 \mathrm{~mm}$ or less compared to maximum unassisted opening, contralateral excursion movements no more than $7 \mathrm{~mm}$, and/or uncorrected deviation to ipsilateral side on mouth opening $[3,7]$. The MRI images reveal disc deformation and its abnormal position within the TMJ $[8,9]$. However, it has been concluded that clinical diagnostic criteria are a reliable method for group IIb diagnosis and not all the patients require MRI before treatment $[10,11]$.

The therapy of closed lock focuses on relieving the pain and normalizing the function of the masticatory system. Different types of methods aim to restore correct position of the disc-condyle complex or adapt joint tissues to new anatomic conditions. The most commonly used primary methods of $\mathrm{Ilb}$ treatment are conservative protocols, e.g., pharmacothera-

*Corresponding author: Marta Miernik, Department of Prosthodontics, Wroclaw Medical University, Krakowska 26, 50-425 Wroclaw, Poland, Tel: +48-71-784-0291, Fax +48-71-784-0292

Accepted: June 01, 2019

Published online: June 03, 2019

Citation: Miernik M, Nowakowska-Toporowska A (2019) Effectiveness of Stretching Exercises in TMJ Closed Lock Management - Case Reports. J Clin Anesth Pain Manag 3(1):66-69 
py, physical therapy, or splint therapy. Surgical methods like arthroscopy or arthroplasty are necessary if conservative methods fail to show any sign of improvement [12,13].

The aim of the study was to present symptomatic treatment of disc displacement without reduction based on stretching exercises, which are easy to implement in every dental practice.

\section{Cases Presentation}

\section{Case 1}

A 65-year-old male was referred from the Department of Oral Surgery to the Department of Prosthetic Dentistry, Wroclaw Medical University, presenting with trismus following a long dental visit. Anamnesis revealed that two weeks before, after the dental visit that was conducted as a preparation for aortic aneurysm surgery, the patient experienced pain in the left TMJ and was unable to open the mouth normally. The patient had no history of TMD or TMJ trauma before this incident. The date of cardiac surgery was postponed as the anesthesiologist reported difficulties with intubation in case of the patient with restricted mouth opening. Establishing physiological range of mandibular opening capacity became crucial for conducting cardiac surgery.

Physical examination showed symptoms of disc displacement without reduction with limited mouth opening (group Ilb RDC/TMD) related to left TMJ. The TMJ was painful upon preauricular palpation. The range of active mouth opening was $22 \mathrm{~mm}$ measured with a ruler with negative result of passive mouth opening test. The deviation of the mandible toward the affected side was observed while opening. Lateral mandibular movements were asymmetrical: left lateral movement had physiological range, but movement toward right side was impossible.

Manipulating the mandible in a downward and forward direction for the reduction of an anteriorly displaced disc brought no clinical effect. It was decided to reestablish normal range of mandible movements by exercise therapy. The massage of masseter and temporal muscles was performed to reduce muscle constriction induced by pain of left TMJ while performing stretching exercises. The protocol of muscle massage included kneading and stretching of the muscles for 15 min. Later, stretching exercises were performed to increase the mobility of the mandible by applying force to open the patient's mouth and overcoming the restricted active movement range. Both operators' forefingers applied force on lower premolars and both thumbs were lying on the upper anterior teeth. The stretchy movements against the resistance were conducted for $5 \mathrm{~min}$ with short breaks. The stretching exercises caused discomfort and pain in the left TMJ.

During the first visit, the achieved range of opening was $26 \mathrm{~mm}$. To accelerate the results of manual therapy, the patient was instructed to perform warming of the left TMJ twice or thrice a day and then stretching exercises on his own at home. The second visit was scheduled for the next day. The active range of opening movement was $25 \mathrm{~mm}$, and after the visit, it was $31 \mathrm{~mm}$. Next visits were scheduled on the third, seventh, eighth, and ninth day after the first visit. During each visit, the same protocol was conducted and progress in mouth opening was observed. On the ninth day, the range of active mouth opening was $36 \mathrm{~mm}$, and after the stretching therapy, it was $42 \mathrm{~mm}$. The last visit was on the twenty-first day since the beginning of the treatment. The patient was able to open his mouth up to $42 \mathrm{~mm}$. With this range of mouth opening, the patient was accepted to undertake intubation for cardiac surgery.

\section{Case 2}

A 15-year-old female visited the Department of Prosthetic Dentistry, Wroclaw Medical University, complaining of pain in the left TMJ and limited mouth opening, which appeared suddenly one week earlier. The patient reported the presence of clicking in the left TMJ for about half of a year before the incident of blockade in the left joint, which appeared while yawning.

Clinical examination revealed left disc displacement without reduction with limited mouth opening (group IIb RDC/ TMD) with the following typical symptoms: Limited mouth opening (18 $\mathrm{mm}$ measured by ruler) with deviation of mandible toward left side, elimination of lateral movement toward right side, and pain in left TMJ.

Manipulating the mandible for the reduction of an anteriorly displaced disc was inefficient. In order to reduce symptoms of IIb RDC/TMD, physical treatment was planned. The patient was undergoing iontophoresis to reduce pain in left TMJ and massage therapy to prevent muscle constriction caused by pain while performing stretching exercises. Considering that the patients' place of residence was far away from the clinic, just three once-a-week physical therapy visits and one control checkup were conducted.

First part of the therapeutic protocol was 15-min iontophoresis with $2 \%$ lidocaine solution to provide analgesic effect in left TMJ. After iontophoresis,a 10-min massage, including kneading and stretching of masseter and temporal muscles, was performed as preparation for stretching exercises. The stretching exercises were conducted as in case 1.

In addition, the patient was instructed on how to perform physical therapy at home. The patient was supposed to warm the area of left TMJ thrice a day, in order to prepare the joint and surrounding muscles for stretching of TMJ. After that three exercises were to be performed. First exercise was the same as conducted in the clinic-stretching TMJ tissues in order to increase the range of mouth opening by using the pressure of forefingers on lower teeth and placing thumbs on upper teeth and applying force to open mouth wider. The aim of the second exercise was to increase the range of lateral movement toward right side. The patient was supposed to roll the wine cork between the upper and lower anterior teeth to practice lateral movements of the mandible. The last exercise was correcting the path of abduction and adduction of the mandible-the patient was supposed to open the mouth along the straight line in front of the mirror.

After the consultation, the patient was advised to perform exercises at home. Two days after the consultation, the range 
of mouth opening was $25 \mathrm{~mm}$, which increased up to $30 \mathrm{~mm}$ after the physical therapy. On the second physical therapy visit, the range of mouth opening increased from 30 to 35 $\mathrm{mm}$. After the third visit, the mouth opening was $37 \mathrm{~mm}$. The patient was recommended to maintain home exercise protocol for the next four weeks after which the control visit was set. The control visit showed that the vertical range (42 $\mathrm{mm}$ ) and path of mandible movement were correct. Also lateral movements were symmetrical. The patient reported no pain in left TMJ.

\section{Discussion}

The chief complaint in presented cases was limited mouth opening. The mouth opening range is a generally accepted measurement of TMJ hypomobility and function. The linear measurement between incisal edges of upper and lower central incisors of $40 \mathrm{~mm}$ is a minimal range considered as physiological mouth opening. Therefore, the aim of the treatment of disc displacement without reduction is the symptomatic improvement of the range of mandibular movement in vertical and horizontal planes $[14,15]$.

The natural course of closed lock is self-improving. Yura's research shows that most of the acute closed locks resolve successfully within 12 weeks [16]. Also in cases of persistent disc displacement, the natural course leads to increase in the intensity of symptoms. Sato, et al. noticed that the incidence of successful resolution was about $34 \%$ at 6 months, $50 \%$ at 12 months, and $68 \%$ at 18 months follow-up [17]. According to Kurita, et al., just one-quarter of joints was still symptomatic in a 2.5-year follow-up study of natural course of closed lock [18]. Although the Ilb disorders seem to be self-limiting, sometimes it is necessary to provide patients with therapy, leading to faster improvement of stomatognathic system function. According to Minakuchi, et al., the improvement in clinical symptoms of closed lock can be achieved by just minimal treatment intervention [19]. The effects of stretching exercises, including movements of mandible in horizontal and vertical planes in described cases, were visible in a short time period.

The studies of Yoshida, et al. show that stretching exercises are an effective method for closed lock management compared to control groups who received no treatment. The effect of mandibular condylar movement exercise (MCME) presented by Yoshida depends on the age and duration of locking-the effect of treatment was present in cases of younger patients with acute closed lock $[20,21]$. Haketa, et al. stated that exercise therapy has better clinical outcomes than splint therapy in IIb disorder management [22]. Also Nicolakis, et al. emphasize that exercise therapy is very effective in case of closed lock management to decrease pain and improve mandible range of motion [23]. However, Craane, et al.'s studies suggested that physical therapy has no additional effect compared to the natural course of closed lock [24].

The self-improvement of clinical symptoms of untreated disc displacement without reduction with limited mouth opening is probably a result of active adaptive reaction of TMJ tissues. The MRI scans show that in cases of Ilb the articular disc becomes more anteriorly displaced and shortened, while condylar height decreases during its natural course, which explains the significant increase in mouth-opening capacity. The same mechanism might be responsible for the success of the described exercises, which also lead to stretching and remodeling of retrodiscal tissues [24-27].

\section{Conclusions}

In the presented cases significant improvements in the range and path of mouth opening were observed. Stretching exercises seem to be an effective symptomatic therapy method for disc displacement without reduction with limited mouth opening in the presented clinical cases.

\section{References}

1. Dworkin SF, LeResche L (1992) Research diagnostic criteria for temporomandibular disorders: review, criteria, examinations and specifications, critique. J Craniomandib Disord 6: 301-355.

2. Poveda-Roda R, Bagán JV, Jiménez-Soriano Y, et al. (2009) Retrospective study of a series of 850 patients with temporomandibular dysfunction (TMD). Clinical and radiological findings. Med Oral Patol Oral Cir Bucal 14: 628-634.

3. Manfredini D, Guarda-Nardini L, Wincour E, et al. (2011) Research diagnostic criteria for temporomandibular disorders: A systematic review of axis I epidemiologic findings. Oral Surg Oral Med Oral Pathol Oral Radiol Endod 112: 453-462.

4. Mariz AC, Campos PS, Sarmento VA, et al. (2005) Assessment of disk displacements of the temporomandibular joint. Braz Oral Res 19: 63-68.

5. de Leeuw R, Klasser GD (2008) Orofacial pain: Guidelines for assessment, diagnosis, and management. Quintessence Publishing, USA.

6. Farrar WB (1978) Characteristics of the condylar path in internal derangements of the TMJ. J Prosthet Dent 39: 319-323.

7. Naeije M, Te Veldhuis AH, Visscher CM, et al. (2013) Disc displacement within the human temporomandibular joint: a systematic review of a 'noisy annoyance'. J Oral Rehabil 40: 139158.

8. Sener S, Akgünlü $F$ (2005) Correlation of different MRI characteristics of anterior disc displacement with reduction and without reduction. J Contemp Dent Pract 6: 26-36.

9. Sener S, Akgünlü $F(2004) M R I$ characteristics of anterior disc displacement with and without reduction. Dentomaxillofac Radiol 33: 245-252.

10. Üşümez S, Öz F, Güray E (2004) Comparison of clinical and magnetic resonance imaging diagnoses in patients with TMD history. J Oral Rehabil 31: 52-56.

11. Emshoff R, Innerhofer K, Rudisch A, et al. (2002) Clinical versus magnetic resonance imaging findings with internal derangement of the temporomandibular joint: An evaluation of anterior disc displacement without reduction. J Oral Maxillofac Surg 60: 36-41.

12. Schiffman EL, Look JO, Hodges JS, et al. (2007) Randomized effectiveness study of four therapeutic strategies for TMJ closed lock. J Dent Res 86: 58-63.

13. Schiffman EL, Velly AM, Look JO, et al. (2014) Effects of four treatment strategies for temporomandibular joint closed lock. Int J Oral Maxillofac Surg 43: 217-226. 
14. Walker N, Bohannon RW, Cameron D (2000) Discriminant validity of temporomandibular joint range of motion measurements obtained with a ruler. J Orthop Sports Phys Ther 30: 484-492.

15. Fricton JR, Schiffman EL (1987) The crandiomandibular index: validity. J Prosthet Dent 58: 222-228.

16. Yura S (2012) Natural course of acute closed lock of the temporomandibular joint. Br J Oral Maxillofac Surg 50: 646-649.

17. Sato S, Kawamura H, Nagasaka H, et al. (1997) The natural course of anterior disc displacement without reduction in the temporomandibular joint: Follow-up at 6, 12, and 18 months. J Oral Maxillofac Surg 55: 234-238.

18. Kurita K, Westesson PL, Yuasa H, et al. (1998) Natural course of untreated symptomatic temporomandibular joint disc displacement without reduction. J Dent Res 77: 361-365.

19. Minakuchi H, Kuboki T, Matsuka Y, et al. (2001) Randomized controlled evaluation of non-surgical treatments for temporomandibular joint anterior disc displacement without reduction. J Dent Res 80: 924-928.

20. Yoshida H, Sakata T, Hayashi T et al. (2011) Evaluation of mandibular condylar movement exercise for patients with internal derangement of the temporomandibular joint on initial presentation. Br J Oral Maxillofac Surg 49: 310-313.

21. Yoshida H, Kashiwagi K, Sakata T, et al. (2013) Prognostic factor of mandibular condylar movement exercise for patients with internal derangement of the temporomandibular joint on initial presentation: preliminary report. J Craniomaxillofac Surg 41: 356-358.

22. Haketa T, Kino K, Sugisaki M, et al. (2010) Randomized clinical trial of treatment for TMJ disc displacement. J Dent Re 89: 12591263.

23. Nicolakis P, Erdogmus B, Kopf A, et al. (2001) Effectiveness of exercise therapy in patients with internal derangement of the temporomandibular joint. J Oral Rehabil 28: 1158-1164.

24. Craane B, Dijkstra PU, Stappaerts K, et al. (2012) Randomized controlled trial on physical therapy for TMJ closed lock. J Dent Res 91: 364-369.

25. Imirzalioglu P, Biler N, Agildere AM (2005) Clinical and radiological follow-up results of patients with untreated TMJ closed lock. J Oral Rehabil 32: 326-331.

26. Cai $X Y$, Jin JM, Yang C (2011) Changes in disc position, disc length, and condylar height in the temporomandibular joint with anterior disc displacement: A longitudinal retrospective magnetic resonance imaging study. J Oral Maxillofac Surg 69: 340-346.

27. Al.-Baghdadi M, Durham J, Steele J (2014) Timing interventions in relations to temporomandibular joint closed lock duration: a systematic review of 'locking duration'. J Oral Rehabil 41: 24-58.

DOI: $10.36959 / 377 / 329$

Copyright: (c) 2019 Miernik M, et al. This is an open-access article distributed under the terms of the Creative Commons Attribution License, which permits unrestricted use, distribution, and reproduction in any medium, provided the original author and source are credited. 